\title{
New Insights into the Biology of Peritoneal Mesothelial Cells: The Roles of Epithelial-to- Mesenchymal Transition and Cellular Senescence
}

\author{
Janusz Witowski ${ }^{a}$ b $\quad$ Krzysztof Książek $^{a} \quad$ Achim Jörres $^{b}$ \\ ${ }^{a}$ Department of Pathophysiology, Poznań University of Medical Sciences, Poznań, Poland; b Department of \\ Nephrology and Medical Intensive Care, Charité-Universitätsmedizin Berlin Campus Virchow-Klinikum, Berlin, \\ Germany
}

\section{Key Words}

Mesothelial cells • Epithelial-to-mesenchymal transition • Senescence $\cdot$ Transforming growth factor- $\beta_{1} \cdot$ Vascular endothelial growth factor $\cdot$ Peritoneal dialysis

\begin{abstract}
Ultrafiltration failure due to dysfunction of the peritoneum as a dialyzing organ is a major clinical limitation of peritoneal dialysis. It is increasingly clear that mesothelial cells play an important role in fibrogenesis and vasculopathy that underlie peritoneal membrane dysfunction. New and extensively studied aspects of peritoneal mesothelial cell biology include epithelial-to-mesenchymal transition and cellular senescence. We discuss the potential significance of these processes for the peritoneal membrane function.
\end{abstract}

Copyright $\odot 2008$ S. Karger AG, Basel

Peritoneal membrane dysfunction and the resulting ultrafiltration failure is the main problem of long-term peritoneal dialysis (PD). It appears to be related to structural alterations that develop in the peritoneum during PD. Typical changes include submesothelial thickening, fibrosis, and increased vascularization, which all are thought to contribute to inadequate transperitoneal ultrafiltration. These processes are believed to be regulated by mediators such as transforming growth factor- $\beta$ (TGF- $\beta$ ) and vascular endothelial growth factor (VEGF) that originate largely from the mesothelium.

\section{KARGER}

Fax +41613061234 E-Mail karger@karger.ch www.karger.com

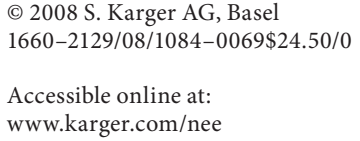

Mesothelial cells line the peritoneal cavity providing a slippery non-thrombogenic surface that protects the viscera and allows their smooth sliding. In addition, mesothelial cells play an important role in solute transport, immune surveillance, inflammation, and wound healing. These functions of mesothelial cells are important also in the context of PD and have thoroughly been discussed in several excellent reviews [1-7]. Numerous studies have shown that both exposure to PD fluid components and the presence of $\mathrm{PD}$-associated inflammation alter the function of mesothelial cells. It is feared that these changes contribute to ultrafiltration failure in PD patients. For that reason, the markers of mesothelial cell integrity and function are frequently assessed to determine the biocompatibility profile of PD fluids [8]. In this minireview we will focus on those new aspects of human peritoneal mesothelial cell (HPMC) biology that are related primarily to its angiogenesis- and fibrosis-modulating activity.

\section{Epithelial-to-Mesenchymal Transition}

Remarkable plasticity and the vasculogenic potential of mesothelial cells can be best seen during development. Embryonic mesothelial cells contribute to the coronary and intestinal vasculature by differentiating into endothelial cells, vascular smooth muscle cells and pericytes $[9,10]$. Adult mesothelia seem to retain much of this differentiative potential [11]. A crucial event in differentia- 
tion processes is epithelial-to-mesenchymal transition (EMT), during which polarized epithelial cells convert in a coordinated sequence of events into fibroblast-like cells capable of locomotion and extracellular matrix (ECM) remodeling. On the other hand, EMT is recognized as a key mechanism in pathological processes, including tumorigenesis and chronic disorders associated with ECM accumulation and fibrosis.

Increasing evidence indicates that EMT occurs in the peritoneal membrane during PD. It appears that in this setting HPMC differentiate into submesothelial fibroblasts and thus contribute to structural and functional alterations of the peritoneum [for a comprehensive review, see 12]. There is some controversy about to whether the desquamated cells found in PD effluent accurately represent the normal HPMC population. Nevertheless, these cells can easily be isolated [3] and their careful examination provides important information about the intraperitoneal milieu. Yanez-Mo et al. [13] have observed that effluent-derived HPMC exhibit different phenotypes that range from the typical epithelial cobblestone-like appearance to the fibroblast spindle-like morphology. They have also found that the presence of fibroblast-like HPMC in the effluent increases with time on PD and occurs more often after episodes of hemoperitoneum and/or peritonitis. Furthermore, they have demonstrated that these cells gradually lose typical cytoskeleton organization and epithelial cell markers (such as E-cadherin and cytokeratins) but progressively upregulate the expression of slug/snail, a zinc-finger transcriptional regulator of EMT. Subsequent analysis of peritoneal biopsies from PD patients revealed the presence of fibroblast-like cells in the submesothelial interstitium [14]. The cells expressed markers typical for the mesothelium and frequently coexpressed $\alpha$-smooth muscle actin ( $\alpha$-SMA) that is typical for activated myofibroblasts. All these observations suggested that fibroblast-like cells in the peritoneum could have originated from HPMC that had undergone EMT.

Molecular mechanisms of EMT have been only partially deciphered [reviewed in 15]. It appears that TGF- $\beta_{1}$ is one of the key mediators of EMT. It has been shown to induce EMT in many cell types, including cultured HPMC [16]. In vivo, adenovirus-mediated overexpression of TGF- $\beta_{1}$ in the rat peritoneum results in peritoneal fibrosis, neoangiogenesis, and increased peritoneal permeability [17]. These processes are associated with the induction of genes involved in EMT (snail, collagen I, $\alpha$ SMA) and the appearance of cytokeratin/ $\alpha$-SMA-positive cells, first in the mesothelium and later in the reorganized submesothelial matrix [18]. Similar alterations have been reported to develop in the peritoneal membrane of uremic rats [19]. They were associated with increased accumulation of advanced glycation end products (AGE) and TGF- $\beta$. The blockade of AGE receptor (RAGE) markedly reduced both TGF- $\beta$ expression and attenuated the EMT-associated alterations. It has also been found that the fibroblast-like phenotype of HPMC isolated from PD patients or treated in vitro with TGF- $\beta_{1}$ could be reversed by bone morphogenic protein-7 (BMP7) [20]. Finally, it appears that EMT-converted HPMC may have an impact on peritoneal vasculature. The effluent-derived HPMC with fibroblast-like morphology were found to secrete more VEGF than HPMC with classic epithelial appearance [21]. Importantly, PD patients from whom these cells were isolated had greater peritoneal permeability and decreased ultrafiltration.

\section{Cell Senescence}

Another cellular response that may potentially be associated with increased fibro- and angiogenic activity is cell senescence. When normal human cells are placed in culture, they can divide only a finite number of times (the Hayflick limit). After that they enter senescence - a state characterized by growth arrest, altered morphology, a different pattern of gene expression, and the presence of senescence markers (e.g. senescence-associated- $\beta$-galactosidase, $S A-\beta-G a l)$. This replicative senescence is caused by progressive telomere shortening and therefore is termed telomere-dependent senescence. The senescence program can also be triggered by other stimuli, such as DNA damage, excessive mitogenic signals, and chromatin disorganization. This response is often termed stressinduced or premature senescence [for a recent comprehensive review, see 22].

Investigators with experience in culturing HPMC have long been aware of the cells becoming senescent relatively quickly. We have investigated this phenomenon in detail and found that, indeed, HPMC developed all typical features of senescence within only few rounds of replication [23]. Surprisingly, HPMC seemed to senesce without telomere shortening [24]. In contrast, senescent HPMC increased the expression of p16, a cyclin-dependent kinase inhibitor involved in telomere-independent senescence [23]. Furthermore, we have detected extensive accumulation of DNA damage foci in senescent cells [24]. The foci can be visualized by the presence of phosphorylated histone $\mathrm{H} 2 \mathrm{AX}(\gamma-\mathrm{H} 2 \mathrm{AX})$ molecules flanking the site of DNA double-strand breaks. In cells that senesce in 
a telomere-dependent manner, $\gamma-\mathrm{H} 2 \mathrm{AX}$ is typically detected within telomeres, which reflects telomere erosion and/or dysfunction. In HPMC, however, $\gamma$-H2AX was predominantly localized in non-telomeric DNA sequences. Altogether, these observations indicate that HPMC in culture senesce prematurely through a telomere-independent mechanism.

Oxidative stress is viewed as one of the main culprits of premature senescence. We have found that senescent HPMC generate more reactive oxygen species (ROS) [23]. This is probably a result of mitochondrial dysfunction, as evidenced by the reduced potential across the mitochondrial inner membrane [24]. In addition, the resources of glutathione (GSH), a major antioxidant, seem to be markedly depleted in senescent HPMC [25]. Therefore, not surprisingly, senescent HPMC display strong presence of 8 hydroxy-2'-deoxyguanosine (8-OH-dG), a marker of DNA oxidative damage [23]. Our preliminary results indicate that antioxidants, when potent and introduced into the culture system early enough, may delay the onset of senescence in HPMC [Książek et al., manuscript submitted].

Interestingly, senescence of HPMC is associated with increased TGF- $\beta_{1}$ release [26]. It appears that increased TGF- $\beta$ activity may underlie at least some aspects of HPMC senescence, including growth arrest, cellular hypertrophy and expression of SA- $\beta$-Gal [26]. We have observed an intriguing relationship between the calendar age of the donor, from whom HPMC were isolated, and the production of TGF- $\beta_{1}$ by these cells in vitro [27]. Cells from older donors were found to secrete more TGF- $\beta_{1}$ and the magnitude of TGF- $\beta_{1}$ release correlated inversely with the life span of HPMC in culture. In this respect, it has recently been reported that HPMC from older donors showed increased expression of pro-inflammatory mediators and increased nuclear factor- $\mathrm{B}(\mathrm{NF}-\kappa \mathrm{B})$-dependent transcriptional activity [28].

Furthermore, senescent HPMC seem to have a different angiogenic activity compared to freshly isolated cells. Conditioned media from senescent HPMC stimulate endothelial cell proliferation to a significantly greater degree compared to media from young HPMC cultures. This effect is probably related to a change in the proportion of angiogenic and angiostatic mediators produced. For example, the expression of early population doubling level cDNA-1/retinal pigmented epithelium-derived factor (EPC-1/PEDF), an angiogenesis inhibitor, decreases in senescent cells [23]. In contrast, the release of VEGF by senescent HPMC significantly increases [29].

While the occurrence of EMT in the peritoneal membrane of PD patients is increasingly well documented, the

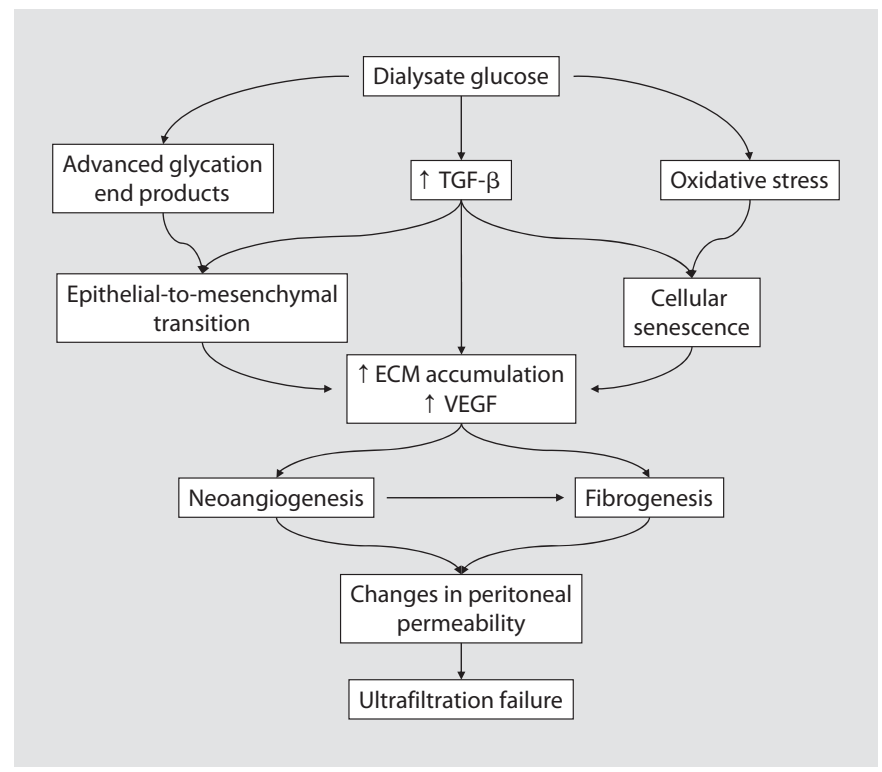

Fig. 1. Hypothetical impact of EMT and cellular senescence on peritoneal remodeling during PD. Exposure of HPMC to dialysate glucose results in increased oxidative stress, increased release of TGF- $\beta_{1}$ and accumulation of AGE. TGF- $\beta_{1}$ and/or AGE may induce EMT which changes HPMC into fibroblast-like cells capable of invading and remodeling the peritoneal interstitium. Increased oxidative stress and TGF- $\beta_{1}$ may contribute to accelerated or premature senescence of HPMC. Both EMT-converted and senescent HPMC may produce increased amounts of ECM, which directly contributes to fibrosis. In addition, such cells may secrete more VEGF, which may lead to neoangiogenesis. Increased blood supply facilitates the progression of fibrosis. The structural alterations lead to peritoneal membrane dysfunction and ultrafiltration failure.

same cannot be said about senescence. This is partly related to technical difficulties in detecting senescent cells in vivo, but also results from our limited knowledge about whether and how cellular senescence might contribute to organismal aging. Gotloib et al. [30] have carefully examined the alterations that develop in mesothelial cells of rats treated intraperitoneally with high loads of glucose. They described the presence of enlarged, senescence-like cells in the mesothelium and suggested that exposure to high glucose led to acceleration of the cell cycle, faster exhaustion of the cell proliferation potential and ultimately cell senescence [31]. In this respect, we have demonstrated that the exposure of HPMC in vitro to high glucose accelerated the onset of cellular senescence, upregulated cell cycle inhibitors p21 and p27, and increased the expression of SA- $\beta-\mathrm{Gal}$ [26]. In addition, glucose increased fibronectin release and exacerbated the hyper- 
trophic phenotype of senescent HPMC. These effects can partly be attributed to the increased generation of ROS and TGF- $\beta_{1}$, as they can be reduced by ROS scavengers and antibodies to TGF- $\beta_{1}[25,26]$.

It remains to be determined whether senescence in the presence of elevated glucose will further increase VEGF secretion. As mentioned earlier, exposure to high glucose results in senescent HPMC releasing more TGF- $\beta_{1}$, and TGF- $\beta_{1}$ per se is known to induce VEGF, at least in earlypassage rat mesothelial cells [32]. On the other hand, however, glucose itself has been found to dose-dependently decrease rather than to stimulate VEGF release by HPMC [33]. In contrast, both glucose degradation products [34] and glycated proteins [33] have been shown to upregulate VEGF synthesis in HPMC.

More recently, Gotloib et al. [35] have reported on the presence of SA- $\beta-$ Gal and p16 in some HPMC isolated from PD effluents. This observation requires further confirmation but may indicate that the exposure to $\mathrm{PD}$ solutions may trigger the senescence response in mesothelial cells in vivo. Although the consequences of such a reaction remain hypothetical, the presence of senescent cells among those shed from the peritoneal surface may potentially impair mesothelial wound healing since freefloating mesothelial cells play an important role in mesothelial recovery after trauma [5]. On the other hand, the accumulation of senescent cells within the mesothelial monolayer could contribute to angiogenesis and fibrosis in the underlying submesothelial tissue, possibly by in- creased secretion of TGF- $\beta_{1}$ and VEGF. Figure 1 is a schematic representation indicating the potential role of EMT and senescence in HPMC biology.

\section{Conclusions}

EMT and cellular senescence are two fundamental biological events, with TGF- $\beta$ implicated as an important mediator in both processes. In addition, TGF- $\beta$ is thought to be involved in the regulation of apoptosis [36] that is an additional crucial axis in cell response to stress and can be viewed as an alternative to senescence [22]. In this respect, it has been shown that TGF- $\beta$ may trigger apoptosis of rat peritoneal mesothelial cells [37], but the regulatory factors that determine whether cells undergo senescence, apoptosis or EMT in response to TGF- $\beta_{1}$ are currently unclear. Thus, the commitment of a cell to a given response appears to depend strictly on the physiological or pathophysiological context. TGF- $\beta$ may therefore serve as a common mediator of distinct pathways with the net biological result depending upon the exact nature of the microenvironment. There is some evidence that HPMC may undergo EMT (and possibly also senescence) in the setting of PD with a resultant increased vasculogenic potential that may further contribute to peritoneal membrane dysfunction. Clearly, more research is still required for better definition of the stimuli and contexts leading to a particular cellular response.

\section{References}

1 Dobbie JW: New concepts in molecular biology and ultrastructural pathology of the peritoneum: their significance for peritoneal dialysis. Am J Kidney Dis 1990;15:97-109.

$\checkmark 2$ Herrick SE, Mutsaers SE: The potential of mesothelial cells in tissue engineering and regenerative medicine applications. Int J Artif Organs 2007;30:527-540.

>3 Lopez-Cabrera M, Aguilera A, Aroeira LS, Ramirez-Huesca M, Perez-Lozano ML, Jimenez-Heffernan JA, Bajo MA, del Peso G, Sanchez-Tomero JA, Selgas R: Ex vivo analysis of dialysis effluent-derived mesothelial cells as an approach to unveiling the mechanism of peritoneal membrane failure. Perit Dial Int 2006;26:26-34.

$\checkmark 4$ Mutsaers SE: The mesothelial cell. Int J Biochem Cell Biol 2004;36:9-16.

\footnotetext{
$\checkmark 5$ Mutsaers SE, Prele CM, Lansley SM, Herrick $>10$ Wilm B, Ipenberg A, Hastie ND, Burch JB, SE: The origin of regenerating mesothelium: a historical perspective. Int J Artif Organs 2007;30:484-494.

6 Raftery AT, Slater ND, Cope GH: Clinical anatomy of the peritoneal mesothelium: a review. Clin Anat 1989;2:69-85.

7 Yung S, Li FK, Chan TM: Peritoneal mesothelial cell culture and biology. Perit Dial Int 2006;26:162-173.

$>$ Krediet RT: Dialysate cancer antigen 125 concentration as marker of peritoneal membrane status in patients treated with chronic peritoneal dialysis. Perit Dial Int 2001;21: 560-567.

9 Munoz-Chapuli R, Gonzalez-Iriarte M, CarBader DM: The serosal mesothelium is a major source of smooth muscle cells of the gut vasculature. Development 2005;132:53175328.

11 Kawaguchi M, Bader DM, Wilm B: Serosal mesothelium retains vasculogenic potential. Dev Dyn 2007;236:2973-2979.

$\checkmark 12$ Aroeira LS, Aguilera A, Sanchez-Tomero JA, Bajo MA, del Peso G, Jimenez-Heffernan JA, Selgas R, Lopez-Cabrera M: Epithelial to mesenchymal transition and peritoneal membrane failure in peritoneal dialysis patients: pathologic significance and potential therapeutic interventions. J Am Soc Nephrol 2007;18:2004-2013.
} mona R, Atencia G, Macias D, Perez-Pomares JM: Cellular precursors of the coronary arteries. Tex Heart Inst J 2002;29: 243-249. 
-13 Yanez-Mo M, Lara-Pezzi E, Selgas R, Ramirez-Huesca M, Dominguez-Jimenez C, Jimenez-Heffernan JA, Aguilera A, SanchezTomero JA, Bajo MA, Alvarez V, Castro MA, del Peso G, Cirujeda A, Gamallo C, SanchezMadrid F, Lopez-Cabrera M: Peritoneal dialysis and epithelial-to-mesenchymal transition of mesothelial cells. N Engl J Med 2003; 348:403-413.

- 14 Jimenez-Heffernan JA, Aguilera A, Aroeira LS, Lara-Pezzi E, Bajo MA, del Peso G, Ramirez M, Gamallo C, Sanchez-Tomero JA, Alvarez V, Lopez-Cabrera M, Selgas R: Immunohistochemical characterization of fibroblast subpopulations in normal peritoneal tissue and in peritoneal dialysis-induced fibrosis. Virchows Arch 2004;444:247-256.

15 Zavadil J, Bottinger EP: TGF- $\beta$ and epithelial-to-mesenchymal transitions. Oncogene 2005;24:5764-5774.

-16 Yang AH, Chen JY, Lin JK: Myofibroblastic conversion of mesothelial cells. Kidney Int 2003;63:1530-1539.

-17 Margetts PJ, Kolb M, Galt T, Hoff CM, Shockley TR, Gauldie J: Gene transfer of transforming growth factor- $\beta_{1}$ to the rat peritoneum: effects on membrane function. J Am Soc Nephrol 2001;12:2029-2039.

-18 Margetts PJ, Bonniaud P, Liu L, Hoff CM, Holmes CJ, West-Mays JA, Kelly MM: Transient overexpression of TGF- $\beta_{1}$ induces epithelial mesenchymal transition in the rodent peritoneum. J Am Soc Nephrol 2005; 16:425436.

$>19$ De Vriese AS, Tilton RG, Mortier S, Lameire NH: Myofibroblast transdifferentiation of mesothelial cells is mediated by RAGE and contributes to peritoneal fibrosis in uraemia. Nephrol Dial Transplant 2006;21:25492555.

-20 Vargha R, Endemann M, Kratochwill K, Riesenhuber A, Wick N, Krachler AM, MalagaDieguez L, Aufricht C: Ex vivo reversal of in vivo transdifferentiation in mesothelial cells grown from peritoneal dialysate effluents. Nephrol Dial Transplant 2006;21:29432947.
21 Aroeira LS, Aguilera A, Selgas R, RamirezHuesca M, Perez-Lozano ML, Cirugeda A, Bajo MA, del Peso G, Sanchez-Tomero JA, Jimenez-Heffernan JA, Lopez-Cabrera M: Mesenchymal conversion of mesothelial cells as a mechanism responsible for high solute transport rate in peritoneal dialysis: role of vascular endothelial growth factor. Am J Kidney Dis 2005;46:938-948.

22 Campisi J, d'Adda di Fagagna F: Cellular senescence: when bad things happen to good cells. Nat Rev Mol Cell Biol 2007;8:729-740.

23 Książek K, Piwocka K, Brzezinska A, Sikora E, Zabel M, Bręborowicz A, Jörres A, Witowski J: Early loss of proliferative potential of human peritoneal mesothelial cells in culture: the role of p16INK4a-mediated premature senescence. J Appl Physiol 2006;100:988995.

24 Książek K, Passos JF, Olijslagers S, Saretzki G, Martin-Ruiz C, von Zglinicki T: Premature senescence of mesothelial cells is associated with non-telomeric DNA damage. Biochem Biophys Res Commun 2007;362: 707-711.

25 Książek K, Bręborowicz A, Jörres A, Witowski J: Oxidative stress contributes to accelerated development of the senescent phenotype in human peritoneal mesothelial cells exposed to high glucose. Free Radic Biol Med 2007;42:636-641.

26 Książek K, Korybalska K, Jörres A, Witowski $\mathrm{J}$ : Accelerated senescence of human peritoneal mesothelial cells exposed to high glucose: the role of TGF- $\beta_{1}$. Lab Invest 2007; 87:345-356

27 Książek K, Winckiewicz M, Staniszewski R, Bręborowicz A, Witowski J: Correlation between the donor age and the proliferative lifespan of human peritoneal mesothelial cells in vitro: is TGF- $\beta_{1}$ a link? Exp Gerontol 2007;42:840-843.

28 Nevado J, Vallejo S, El Assar M, Peiro C, Sanchez-Ferrer CF, Rodriguez-Manas L: Changes in the human peritoneal mesothelial cells during aging. Kidney Int 2006;69:313-322.
29 Książek K, Jörres A, Witowski J: Senescence induces a proangiogenic switch in human peritoneal mesothelial cells. Rejuv Res 2008; in press.

30 Gotloib L, Shostak A, Wajsbrot V, Kushnier R: High glucose induces a hypertrophic, senescent mesothelial cell phenotype after long in vivo exposure. Nephron 1999;82: 164-173.

31 Shostak A, Wajsbrot V, Gotloib L: High glucose accelerates the life cycle of the in vivo exposed mesothelium. Kidney Int 2000;58: 2044-2052.

32 Szeto CC, Lai KB, Chow KM, Szeto CY, Wong TY, Li PK: Differential effects of transforming growth factor- $\beta$ on the synthesis of connective tissue growth factor and vascular endothelial growth factor by peritoneal mesothelial cell. Nephron Exp Nephrol 2005; 99:e95-e104.

33 Mandl-Weber S, Cohen CD, Haslinger B, Kretzler M, Sitter T: Vascular endothelial growth factor production and regulation in human peritoneal mesothelial cells. Kidney Int 2002;61:570-578.

34 Leung JC, Chan LY, Li FF, Tang SC, Chan KW, Chan TM, Lam MF, Wieslander A, Lai KN: Glucose degradation products downregulate $\mathrm{ZO}-1$ expression in human peritoneal mesothelial cells: the role of VEGF. Nephrol Dial Transplant 2005;20:13361349.

35 Gotloib L, Gotloib LC, Khrizman V: The use of peritoneal mesothelium as a potential source of adult stem cells. Int J Artif Organs 2007;30:501-512.

36 Siegel PM, Massague J: Cytostatic and apoptotic actions of TGF- $\beta$ in homeostasis and cancer. Nat Rev Cancer 2003;3:807-821.

37 Szeto CC, Chow KM, Lai KB, Szeto CY, Kwan BC, Li PK: Connective tissue growth factor is responsible for transforming growth factor- $\beta$-induced peritoneal mesothelial cell apoptosis. Nephron Exp Nephrol 2006;103: e166-e174. 